\title{
Corpus
}

\section{Stylèmes et corpus génériques : un essai de confrontation au service de la stylistique des}

genres

\section{Alain Guyot}

\section{CpenEdition}

\section{Journals}

Édition électronique

URL : http://journals.openedition.org/corpus/472

DOI : $10.4000 /$ corpus. 472

ISSN : 1765-3126

Éditeur

Bases; corpus et langage - UMR 6039

Édition imprimée

Date de publication : 1 décembre 2006

ISSN : 1638-9808

Référence électronique

Alain Guyot, "Stylèmes et corpus génériques : un essai de confrontation au service de la stylistique des genres », Corpus [En ligne], 5 | 2006, mis en ligne le 29 juin 2007, consulté le 07 septembre 2020. URL : http://journals.openedition.org/corpus/472 ; DOI : https://doi.org/10.4000/corpus.472

Ce document a été généré automatiquement le 7 septembre 2020

(c) Tous droits réservés 


\title{
Stylèmes et corpus génériques : un essai de confrontation au service de la stylistique des genres
}

\author{
Alain Guyot
}

1 Quelle influence l'appartenance d'une œuvre à un genre littéraire donné peut-elle avoir sur le style de celle-ci? L'existence d'un "cahier des charges » générique peut-elle susciter l'apparition de stylèmes caractéristiques, non point seulement d'un auteur, mais aussi d'un genre littéraire? Face à un même objet, un écrivain peut-il avoir des choix d'écriture différents en fonction des contraintes induites, explicitement ou non, par le genre et sa réception par le public? Ces questions ont, on le sait, fait l'objet d'âpres débats depuis que Pierre Larthomas, dans un article resté célèbre, a réhabilité la notion de genre littéraire en stylistique. Si la rhétorique des genres a marqué le pas depuis le milieu du XIXe siècle, il serait toutefois bien hasardeux de vouloir s'intéresser au style d'une œuvre sans se poser au moins la question des contraintes élémentaires qui président à sa composition et à sa réception. Comme le résume nettement Karl Cogard,

qu'il le veuille ou non, l'écrivain se situe toujours dans une pratique discursive et collective. [...] Dès qu'il y a prise de parole - littéraire ou non -, il y a des contraintes, discursives ou génériques, à prendre en compte [...]. L'analyse stylistique ne peut donc se passer d'une interrogation préalable sur le genre adopté. (p. 117)

1. Existe-t-il des « stylèmes de genre »?

2 Une telle démarche ne vise pas, bien entendu, à rétablir le carcan des genres traditionnels et les clivages souvent stériles qu'ils entraînaient, mais bien plutôt à situer le problème en amont même de la notion de genre littéraire, comme y invite Pierre Larthomas lui-même à travers l'opposition qu'il suggère entre le dit et l'écrit pour mieux cerner les spécificités du langage dramatique ${ }^{1}$. Une telle position permet en effet d'éviter la mise en question de la notion même de genre littéraire qui se fait jour

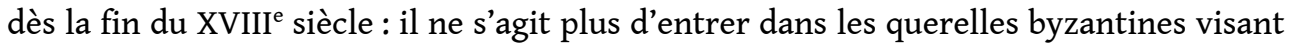
à définir a priori ce qui fait, par exemple, l'essence de la comédie et celle de la tragédie, 
mais de s'interroger sur les conditions d'énonciation et de réception d'une œuvre à partir du moment où, bon gré mal gré, elle s'inscrit dans un genre littéraire donné, si lâche soit-il. Ce sont en effet ces conditions mêmes qui font qu'un auteur va utiliser certains procédés de langage, d'abord en vue d'une certaine efficacité, mais aussi en fonction des attentes de son public, attentes dont il ne peut manquer d'avoir conscience et qui sont elles aussi soumises au genre littéraire sous les auspices duquel l'œuvre s'inscrit. On pourrait alors s'interroger sur la possibilité d'isoler des stylèmes caractéristiques de ces conditions bien particulières d'énonciation et de réception qu'entraîne l'inscription d'une œuvre dans un genre littéraire donné. Encore faudrait-il que cette enquête ne parte pas d'une position a priori fondée sur les invariants du genre - position qui serait celle du poéticien -, mais de l'analyse d'un corpus de textes donné - en l'occurrence ceux qui se rattachent à ce genre littéraire -, analyse susceptible de prendre en compte les phénomènes de variation et qui pourrait alors amener à engager une réflexion sur l'articulation entre corpus et stylistique.

3 Une manière possible d'envisager cette délicate question pourrait procéder d'une confrontation entre différentes versions d'un même motif abordé par un même auteur dans des genres littéraires différents : le traitement du motif au sein d'une œuvre varie$\mathrm{t}$-il en fonction du genre dans lequel elle s'inscrit? Quels indices d'ordre strictement stylistique peut-on retirer de la confrontation entre les versions, indices dont l'analyse permettrait de dégager et de construire les spécificités et les contraintes propres aux genres littéraires étudiés ? Encore faut-il se garder d'élaborer semblable étude à partir d'un seul auteur, dont la manière tout individuelle d'affronter les contraintes génériques risquerait de susciter des phénomènes d'inférence dans les analyses et, partant, dans les conclusions que l'on souhaiterait tirer à propos des genres littéraires approchés. Il convient donc de définir une gamme de genres dans lesquels une pareille confrontation soit envisageable - ce qui parait relever de la gageure, surtout lorsque l'on tient compte du contexte socio-culturel dans lequel ils s'inscrivent...

4 Peut-on alors définir un corpus de textes de genres littéraires différents, mais comparables, communs à un certain nombre d'auteurs appartenant eux-mêmes à une période donnée au cours de laquelle les contraintes spécifiques aux genres envisagés ne soient pas susceptibles de variations majeures, mais permettent en même temps de saisir, sur une échelle chronologique relativement brève, les moments forts d'une évolution? Il se trouve que l'histoire littéraire fournit une matière d'une richesse inestimable, qui a servi de base à une étude dont on souhaiterait présenter ici les prémisses, les objectifs et les modes opératoires, mais aussi les inévitables difficultés qu'elle a pu rencontrer et quelques-unes des pistes qu'elle est parvenue à dessiner.

2. Un corpus : les descriptions issues des voyages d'écrivains aux XVIII et $\mathrm{XIX}^{\mathrm{e}}$ siècles

5 On sait bien en effet que les bouleversements intervenus à la fin des Lumières dans les idées, les mentalités et la sensibilité artistiques déterminent chez les écrivains un intérêt nouveau pour les spectacles du monde, qui les incite à se dégager du carcan de la tradition, en particulier lorsqu'il s'agit pour eux de représenter paysages et objets. Le locus amoenus, indépassable modèle de la description paysagère depuis Homère, semble dans ces conditions sérieusement remis en cause au profit d'une représentation où l'observation, l'originalité et la nouveauté auraient une meilleure part. De même, les objets, les vêtements ne sont plus de simples instruments à disposition : ils acquièrent peu à peu un poids de signification dans leur rapport à l'énonciateur ou au personnage qui trouvera sa pleine expression dans le roman du $\mathrm{XIX}^{\mathrm{e}}$ siècle $^{2}$. L'expression de la 
«libre nature », l'authenticité de la «chose vue », l'originalité de l'impression qu'elle suscite et dans laquelle s'inscrit un point de vue subjectif deviennent progressivement des critères plus décisifs, aux yeux des tenants de l'idéologie littéraire, que les variations aussi virtuoses que respectueuses sur les conventions des topoï traditionnels, qui cherchaient avant tout à manifester un savoir-faire rhétorique à l'intérieur d'un cadre préalablement défini. C'est probablement ce qui amène, pour la première fois peut-être dans l'histoire de la littérature, un certain nombre d'écrivains de renom à voyager avec l'écriture pour principal objectif. Si en effet les périples d'un Bougainville et même d'un Bernardin de Saint-Pierre sont encore fortement motivés par l'enquête scientifique ou philosophique, dans la droite ligne des Lumières, ceux d'un Chateaubriand, d'un Lamartine ou d'un Gautier s'attachent plus nettement à la réalisation d'un véritable projet littéraire: "rapporter des images» à l'instar de Chateaubriand ou entreprendre une œuvre.

6 La riche matière glanée au cours de ces voyages se retrouve bien entendu dans les notes, les carnets et les journaux de ces écrivains. Mais - ce qui ne manque pas d'intéresser notre propos -, un même auteur la réexploite bien souvent dans l'ensemble de son œuvre littéraire, d'un ouvrage à l'autre, sous la forme de descriptions. Qu'il s'agisse de récits de voyage, de romans et autres fictions narratives, de poèmes, d'essais philosophiques ou d'écrits autobiographiques, l'œuvre d'un écrivain est ainsi susceptible d'offrir des descriptions de paysages, d'objets, de personnages ou d'actions identiques d'un point de vue référentiel. Dans ces conditions, la confrontation de ces représentations littéraires d'un même objet dans des ouvrages appartenant à des genres littéraires différents chez un même auteur fait-elle apparaître des variations dans l'écriture de celui-ci, et, si tel est le cas, comment interpréter ces variations? Correspondent-elles à des choix littéraires qui seraient fonction de la visée propre à un écrivain soucieux d'efficacité stylistique, ou bien de contraintes spécifiques auxquelles celui-ci il doit se soumettre en fonction du genre littéraire dans lequel il inscrit son projet? Des confrontations du même type menées chez différents auteurs, et livrées à une analyse stylistique rigoureuse, sont-elles à même de mettre en lumière des variations assez significatives pour révéler des traits pertinents et caractéristiques des stylèmes - propres aux genres littéraires en question, à leur manière d'aborder les objets représentés et d'envisager l'insertion comme l'esthétique de la description littéraire en leur sein? Une confrontation de ce type pourrait parfaitement s'inscrire dans le projet de construction d'une stylistique des genres littéraires envisagé au début de cette étude - ne serait-ce qu'en contribuant à désinféoder la description de son objet, autrement dit de la perspective référentielle qui en a trop souvent gêné l'étude.

3. Questions de méthode3.1. Limites chronologiques

7 Reste qu'un tel projet ne peut se construire que sur la base d'un corpus rigoureusement élaboré, et c'est là que se posent les questions méthodologiques les plus délicates. Comment constituer en effet ce corpus ? Il faut peut-être commencer par une définition de la période envisagée, une période suffisamment cohérente pour éviter, comme on l'a déjà dit, que les variations du contexte socio-culturel n'aient trop d'influence sur les genres littéraires envisagés. Le terminus a quo a dans ce cas été défini précédemment : il s'agit de la fin des Lumières, qui ouvre le champ à la multiplication des expériences existentielles et littéraires - en particulier au voyage et à son récit. Un écrivain comme Bernardin de Saint-Pierre, auteur d'un très atypique Voyage à l'île de France (1773) qui ouvre le chemin au récit de voyage romantique et résonne dans l'œuvre entière, de Paul 
et Virginie aux Etudes et aux Harmonies de la nature, pourrait représenter une sorte de point de départ, sinon de modèle, pour l'étude que l'on entend mener. Le terminus ad quem risque d'être plus difficile à déterminer, la vogue des voyages d'écrivains se déroulant tout au long du XIX ${ }^{\mathrm{e}}$ siècle. On peut toutefois établir que la Première Guerre Mondiale met un terme au voyage à vocation descriptive, duquel l'écrivain rapportait force tableaux pour son public: le voyage sera désormais politique, ethnologique, existentiel, sans que l'œuvre littéraire en soit nourrie de manière systématique. On pourrait donc voir chez un Loti, un Claude Farrère ou un Segalen le point d'aboutissement du corpus à définir - d'autant que c'est le même Loti qui fait apparaître le plus nettement l'évolution des genres issus de ces périples (récit de voyage, autobiographie, poésie, essai, roman) par l'effacement presque total des frontières qui subsistaient encore entre eux.

3.2. Limites génériques

8 Une autre contrainte tient à la nature des genres littéraires susceptibles de faire l'objet d'une confrontation. Il est en effet tentant de mettre en regard les descriptions issues des notes préliminaires, accumulées pendant le voyage, et leur récriture dans les ouvrages explicitement destinés à la publication, mais ces notes, qui ne l'étaient pas, peuvent-elles être retenues dans la perspective qui est la nôtre? On peut certes considérer le journal de Jérusalem de Chateaubriand, les carnets de voyage de Mme de Staël, les notes placées par Flaubert, en guise de sommaires, en tête des différents chapitres de Par les Champs et par les grèves, ou même celles prises par Fromentin à l'île de Ré, comme autant d'avant-textes d'œuvres qui relèvent pour leur part bel et bien de genres institutionnels, qu'il s'agisse de l'Itinéraire, des Martyrs, de Corinne, d'Un cœur simple ou de Dominique. Mais ces notes, par leur nature même, relèvent du champ privé : elles n'ont - à cette période du moins - pas été écrites pour être publiées, ce qui leur ôte toute la dimension collective propre à la réception publique de l'œuvre littéraire ${ }^{3}-$ si importante, on l'a vu, pour comprendre les enjeux d'un genre. La confrontation paraît dans ce cas relever plutôt de la génétique et, si elle permet d'interroger le style de l'écrivain, n'offre que bien peu d'éléments pour cerner la notion de genre littéraire en stylistique.

9 Il faut également prendre garde au fait que les écrivains dont les ouvrages sont susceptibles d'entrer dans le champ de l'analyse ont touché le plus souvent à de nombreux genres, mais rarement aux mêmes. Si, à la suite de leurs courses, Bernardin, Chateaubriand, Hugo et Gautier, par exemple, ont tous écrit des récits de voyage et des fictions narratives, les deux premiers se sont également frottés à l'essai philosophique, tandis que les deux derniers s'orientaient résolument vers la poésie, Chateaubriand se réservant en outre la voie de l'autobiographie. Il est certes possible, dans ces conditions, de tirer des enseignements sur la poétique descriptive de ces différents auteurs, mais dans la perspective générique qui doit être la nôtre, les conclusions risquent d'être d'autant plus limitées, sinon hasardeuses, que les genres abordés seront plus hétérogènes... Il conviendrait donc, dans un premier temps au moins, de se limiter à des genres convenablement représentés dans un corpus suffisamment large, et présentant un certain nombre de parentés formelles offrant prise à la confrontation. Il se trouve que le roman et ses avatars, d'une part, le récit de voyage, de l'autre, genres narratifs par essence, auxquels la plupart des auteurs se sont essayés, ont avec la description des relations certes fort différentes, mais tout aussi essentielles, auxquelles l'analyse pourrait s'attacher avec profit. 
10 Encore faut-il s'entendre sur les concepts de «roman» - ou, plus largement, de "fiction narrative» - et de "récit de voyage»: peut-on, dans ce cas, véritablement parler de "genre littéraire»? La fiction narrative regroupe en effet, outre le roman, des formes aussi diverses que la nouvelle, le conte, l'histoire, la pastorale, l'épopée, voire le récit de voyage imaginaire. Quant au récit de voyage à proprement parler, il paraît à première vue difficile de mettre dans un même ensemble les projets hétérogènes, voire contradictoires, d'un Chateaubriand ou d'un Bernardin et les formes très ambiguës proposées par un Nerval, un Fromentin ou un Loti. Et d'ailleurs, à quel genre rattacher le Voyage en Orient ou Le Désert, Un Eté dans le Sahara ou Madame Chrysanthème? La frontière entre le récit d'un voyage réel et sa mise en fiction narrative est plus que ténue, si tant est qu'après tout, un récit de voyage destiné à la publication soit autre chose qu'une fiction narrative... Les conclusions pour le moins modérées que Gérard Genette tire de l'enquête qu'il a menée sur «l'hypothèse d'une différence a priori de régime narratif entre fiction et non-fiction $»^{4}$ ne peuvent que nous inciter à la prudence en ce domaine : il est en effet bien forcé de constater que les relations entre les deux régimes se caractérisent, du point de vue énonciatif, par d'innombrables emprunts, échanges et contaminations qui en rendent la frontière particulièrement poreuse et que

le «discours de fiction» est en fait un patchwork, ou un amalgame plus ou moins homogénéisé d'éléments hétéro-clites empruntés pour la plupart à la réalité5. (1991, p. 60)

11 Mais s'il met en cause l'opposition ontologique traditionnelle entre récit « fictionnel » et récit «factuel», il refuse tout autant d'abandonner le principe d'une "enquête empirique " susceptible d'élaborer des modèles a posteriori, ce qui rejoint parfaitement le point de vue que nous essayons de soutenir. Si l'on se place en effet, à l'instar de Pierre Larthomas, en amont d'une caractérisation générique a priori du récit de voyage et de la fiction narrative, il est évident qu'un certain nombre de valeurs réunissent les différents sous-ensembles formels que l'on a coutume de réunir sous l'une ou l'autre de ces appellations. Comme l'affirme justement Daniel Sangsue,

[q]ue le roman n'ait pas été patronné théoriquement ne signifie pas pour autant qu'il soit condamné à l'informité et au dérèglement ${ }^{6}$,

et il est fort vraisemblable que l'on puisse trouver le même type de position au sujet du récit de voyage. Le statut rhétorique relativement flou de ces formes narratives ne nuit nullement à leur efficacité littéraire, d'autant que le public ne recevra pas de la même façon un texte selon l'étiquette générique sous laquelle il se présente. Les attentes qu'il aura à l'égard d'un ouvrage se présentant comme un récit de voyage, si mensonger soitil, ne seront jamais les mêmes que ce qu'il souhaitera trouver dans une fiction narrative, quelque parenté qu'elle puisse avoir avec une forme véridictionnelle, et l'auteur sera bien forcé d'en tenir compte. Il appartiendra donc à l'analyse de mettre en lumière les caractères communs à chacun de ces ensembles génériques, tout en examinant les stratégies qu'ils développent à l'égard de la description.

\subsection{Ampleur et validité dans le choix du corpus de référence}

13 Cela posé, il reste à déterminer le corpus d'ouvrages sur lequel devra porter l'enquête. Là encore, les choses ne sont pas simples. Les auteurs ayant publié, à la suite de leurs périples, des récits de voyages et des fictions narratives sont certes légion entre la fin $\mathrm{du} \mathrm{XVIII}^{\mathrm{e}}$ siècle et le début $\mathrm{du} \mathrm{XX}^{\mathrm{e}}$ siècle. On n'évoquera, outre les écrivains déjà cités, que les noms de Nodier, Stendhal, Custine, Sand, Gautier, Mérimée, Dumas, Du Camp, 
les Goncourt ou Gobineau, entre autres, sans même compter les nombreux romanciers populaires qui furent également des voyageurs, à l'instar de ce Ferry, qui rapporta d'un périple en Amérique latine des Scènes de la vie mexicaine parues entre 1846 et 1849 dans la Revue des Deux Mondes, ainsi que deux romans d'aventures publiés dans les années 1850 : Coureur des bois et Costal l'Indien ${ }^{7}$. Mais cela ne signifie pas pour autant que l'on dégagera chez chacun de ces auteurs un corpus significatif de textes à confronter : des auteurs aussi peu « descriptifs » que Stendhal, Mérimée ou Dumas ne peuvent offrir, en dépit de leur abondante production romanesque et viatique, matière à une analyse autre qu'anecdotique. De même, si le Gautier qui parcourt la Belgique ou l'Espagne, et écrit par la suite La Toison d'or ou Militona, qui semblent parfois des décalques des récits de voyage correspondants, fournit au stylisticien un corpus de confrontation inestimable, il n'en est pas de même de l'auteur d'Italia et des nouvelles napolitaines, puisque le récit du voyage en Italie s'interrompt à l'issue du séjour de l'écrivain à Florence et ne se voit repris qu'à l'occasion de la description des Cascine dans Avatar... La relative hétérogénéité générique, en particulier au sein de la fiction narrative (pastorale chez Bernardin, épopée chez Chateaubriand, roman ou nouvelle chez Gautier, conte chez Flaubert ou Nodier, entre autres), se double donc d'un relatif déséquilibre entre les auteurs du corpus de référence, qui amènera peut-être à se concentrer, dans un premier temps au moins, sur des écrivains et des ouvrages offrant la matière d'une confrontation significative. Le travail qui sert de référence à cette étude s'est ainsi essentiellement consacré à des confrontations entre des extraits d'ouvrages des derniers auteurs cités.

\subsection{Choix du corpus d'étude et des outils d'investigation}

14 Reste bien entendu la question du choix des extraits à confronter et des outils indispensables à une investigation efficace. On sait, depuis les récents travaux de Philippe Hamon ou Jean-Michel Adam et les polémiques par eux suscitées ${ }^{8}$, que la description est loin d'être un objet simple à définir et à analyser, et l'on n'y reviendra pas ici en détail. Il faut se souvenir en outre que, chez un même auteur, un morceau descriptif est souvent, dans un ouvrage, élaboré à partir de plusieurs passages d'un autre ouvrage, ou que telle description, ici unique, se retrouve multipliée ailleurs. Il importe donc de se doter d'un certain nombre d'instruments suffisamment simples et généraux pour aborder des textes protéiformes, tout en protégeant la démarche des schémas rigides ou des modèles théoriques trop sophistiqués, susceptibles d'en prédéterminer les conclusions. C'est pourquoi on peut fonder la recherche sur le modèle du "système descriptif » mis en place par Ph. Hamon et sur quelques axes d'analyse empruntés à de récents travaux en la matière ${ }^{9}$ : marques internes ou externes signalant les descriptions dans le contexte, ici narratif - on s'intéressera alors plus précisément aux signes distinctifs entre description et narration -, modes d'organisation interne des éléments descriptifs, fonctions et distribution des passages descriptifs dans le récit.

4. Un exemple d'analyse : le traitement de l'analogie

Il est à la fois difficile et un peu artificiel de prétendre résumer en quelques pages les résultats d'une longue recherche. Avant de proposer les conclusions auxquelles on a pu aboutir sur la base d'un examen détaillé du corpus sélectionné et des outils méthodologiques utilisés, on voudrait tout de même les fonder sur l'analyse de quelques traits révélateurs, en les illustrant d'exemples. D'un point de vue stylistique, on a pu dégager en effet un certain nombre de procédés dont les deux genres considérés font un usage significatif. Jouant en effet sur les particularités d'une syntaxe 
descriptive fondée sur le parallélisme et l'empilement, ceux-ci les combinent avec les possibilités qu'offre toute description de déployer un lexique d'une richesse ou d'une profusion inaccoutumées, suscitant des effets de liste ou des jeux sur le signifiant plus difficilement compatibles avec d'autres gammes de discours. Il suffit de penser ici à Bernardin ou à Chateaubriand, qui se contente le plus souvent d'accumuler des termes empruntés au lexique botanique ou vernaculaire pour créer l'effet "exotique » si particulier aux descriptions de leurs récits de voyage, de Paul et Virginie, d'Atala ou des Natchez. Gautier aime à pratiquer lui aussi ce type d'association entre lexique et syntaxe, dans son Voyage en Espagne comme dans Militona, le roman qui lui fait pendant. De même, les deux genres n'hésitent pas à utiliser toutes les ressources des figures de l'analogie (comparaison, métaphore), ainsi qu'un certain nombre de procédures démarcatives destinées tantôt à mettre en valeur, tantôt à naturaliser la description dans son contexte ${ }^{10}$.

Toutefois tous ces stylèmes descriptifs désormais bien répertoriés ${ }^{11}$ sont souvent mis en œuvre dans les récits de voyage et les fictions narratives selon des visées et des modes différents, et l'on rejoint ici les intuitions de P. Larthomas ${ }^{12}$. C'est en effet au sein du genre et du régime narratif particulier dans lequel s'inscrit son ouvrage que chaque auteur, en fonction de son projet particulier, s'efforce d'affronter et de résoudre, avec plus ou moins de bonheur, les difficultés posées par l'insertion ou le développement de ses descriptions. On souhaiterait ici examiner avec une particulière attention la manière fort révélatrice dont chaque écrivain aborde la question de l'analogie en fonction du genre auquel il se soumet.

\subsection{Comparaisons et métaphores}

Philippe Hamon a bien montré en effet le rôle essentiel de l'analogie dans les systèmes descriptifs ${ }^{13}$. De plus, avant même d'être une catégorie rhétorique, elle constitue un des modes d'écriture privilégiés du récit de voyage, au moins dans sa dimension cognitive. Sous sa forme "sérieuse ", celui-ci a en effet pour objectif de recenser et de présenter, de la manière la plus rationnelle et en même temps la plus "évidente ", une réalité différente de celle dans laquelle évoluent le voyageur-narrateur-descripteur et le public auquel il s'adresse $\mathrm{e}^{14}$. Lorsqu'il s'agit de passer à la description de cette réalité, le voyageur va donc se retrouver confronté à la difficulté de rendre sous la forme de signes l'altérité radicale et à première vue "indescriptible » de cette réalité étrangère et absente : l'analogie se présente alors comme un des moyens les plus efficaces pour réduire cette altérité ${ }^{15}$. Comparaison et métaphore peuvent donc apparaître comme deux modes privilégiés de l'écriture descriptive, dans le récit de voyage comme dans la fiction narrative, mais une étude attentive menée sur les ouvrages de Bernardin, Chateaubriand et Gautier semble marquer une préférence assez nette du premier genre pour la comparaison et du second pour la métaphore. On se contentera de quelques exemples, parmi tant d'autres. Le premier est emprunté à la description de la tempête qui a fait la gloire de Bernardin : 


\begin{tabular}{|c|c|c|}
\hline $\begin{array}{c}\text { Voyage à l'vle de } \\
\text { Fronce }\end{array}$ & manuscrit & Paulet Virginie \\
\hline $\begin{array}{l}\text { Le rivage battu des flots } \\
\text { était couvert d'une } \\
\text { mousse blanche comme 1a } \\
\text { neige, qui s'y entassait } \\
\text { comme des ballots de } \\
\text { coton. (Y. Bénot éd., } \\
\text { Paris, Maspéro-La } \\
\text { Découverte, 1983, p. 184) }\end{array}$ & 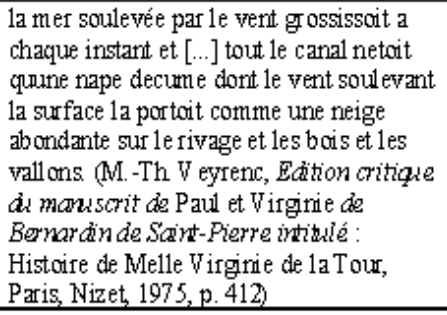 & $\begin{array}{l}\text { La mer, soul evée par le vent, grossissait à chaque } \\
\text { instant, et toutle canal [...] n'était qu'une vaste } \\
\text { nappe d'écumes blanches, creusées de vagues } \\
\text { noires et profondes. Ces écumes s'am assaient } \\
\text { dans le fond des anses [...]. A leurs flocons blancs } \\
\text { et inmombrables, qui étaient chassés } \\
\text { horiz ontalem ent jusqu'au pied des montagnes, on } \\
\text { eût dit d'une neige qui sortait de la mer. (J. Ehrard } \\
\text { éd., Paris, Gallimard, } 1984, \text { p. } 222-223 \text { ) }\end{array}$ \\
\hline
\end{tabular}

Nous avons adjoint, pour la clarté de la comparaison, l'une des versions primitives du passage, conservée par le manuscrit de la bibliothèque Victor-Cousin. Le travail de l'analogie est ici évident: là où le récit de voyage se contente d'une comparaison aux allures de cliché (une mousse blanche comme la neige), suivie immédiatement d'une autre (comme des ballots de coton), certes plus originale dans le rendu impressif, mais en totale rupture tonale avec la précédente, le roman tâtonne au contraire à la recherche d'une véritable image, susceptible de rendre à la fois la réalité du phénomène et l'émotion ressentie par le descripteur. C'est sans doute pour cette raison que Bernardin choisit de disjoindre nettement les deux analogies (la nappe d'écumes / la neige) et d'offrir à la seconde un véritable processus de lancement, sous la forme d'une structure syntaxique (A leurs flocons..., on eût dit...) qui met en place le champ lexical destiné à soutenir et remotiver la comparaison (flocons blancs et innombrables, montagne), et qui recule au maximum l'apparition du comparant en l'isolant afin de lui conférer toute sa valeur poétique. Celle-ci est d'ailleurs renforcée par l'allitération du phonème [7] et le rythme - presque parfait - d'un alexandrin. Outre le travail sur l'analogie auquel se livre Bernardin, on notera au passage une certaine dérive de la comparaison primitive vers la métaphore par l'effacement de l'outil de comparaison (... n'était qu'une nappe...) ou sa modalisation (on eût dit), tandis que la comparaison ainsi modalisée contient elle-même une métaphore (une neige qui sortait de la mer).

Du côté de Chateaubriand, fidèle disciple de Bernardin pour le rendu exact de réalité propre à ses récits de voyage, on est frappé, hormis quelques traits propres à l'écriture épique, par la fréquente transformation des comparaisons en métaphores dans les récits de fiction correspondants : 


\begin{tabular}{|c|c|}
\hline Voyage en Amérizue & Atola \\
\hline $\begin{array}{l}\text { [à propos de la crue printarière du } \\
\text { Missi ssipi] A cette époque, l'e eal } \\
\text { s'élève à une grande hauteur, noie ses } \\
\text { rivages, et ne retourne point aufleuve } \\
\text { dont elle est sortie, comme l'eau du } \\
\text { Nil [...]. (Gorvres romanesques et } \\
\text { voyages, M. Regard éd. Paris, } \\
\text { Gallimard. } 1969 \text {, t. I, p. } 722-723 \text { ) }\end{array}$ & $\begin{array}{l}{[\ldots] \text { c'est le Nil des déserts. (Colvres }} \\
\text { romanesques et voyages, op. cit., t. I, } \\
\text { p. 34) }\end{array}$ \\
\hline $\begin{array}{l}\text { Voilà l' orage ! Qu'on se figgre un } \\
\text { déluge de feu sans vent et sans ealu; } \\
\text { l'odeur de sufre remplit l'air ; la } \\
\text { nature est éclairée comme à la lueur } \\
\text { d'un embrasement. } \\
\text { [...] } \\
\text { Les Indiens disent que le bruit du } \\
\text { tonnerre est causé par des oiseaux } \\
\text { immenses qui se battent dans l'air, et } \\
\text { par les efforts que fait un vieillard } \\
\text { pour vomir une couleure de feu. En } \\
\text { preuwe de cette assertion, ils montrent } \\
\text { des arbres où la foudte a tracél'im age } \\
\text { d'un serpent. Souvent les orages } \\
\text { mettent le feu aux forêts; ; elles } \\
\text { contimuent de brûler juscu'à ce que } \\
\text { l'incendie soit arrêté par le cours de } \\
\text { quelque fleuve [.... (ibid, p. 733) }\end{array}$ & 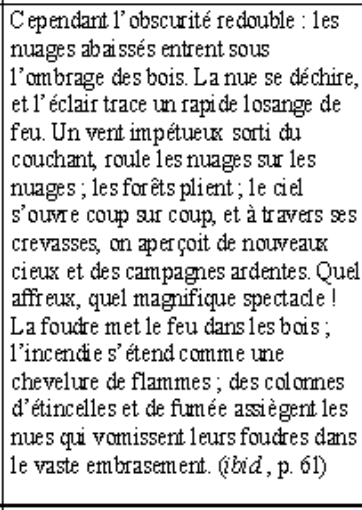 \\
\hline Itinéraire & Les Mortyrs \\
\hline $\begin{array}{l}\text { L'eau du fleuve [le Nil] était dans cet } \\
\text { endroit d'un rouge tir ant sur le violet, } \\
\text { de la coul eur d'une bruyère en } \\
\text { automne. (Gorvres romanesques er } \\
\text { voyages, op. cit., t. II, p. 1134) }\end{array}$ & 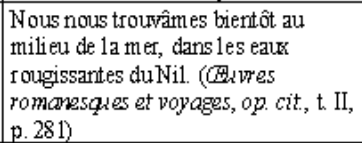 \\
\hline
\end{tabular}

$\mathrm{Si}$, dans le cas de l'orage, on peut mettre la métaphorisation presque excessive de l'extrait d'Atala sur le compte de l'instance d'énonciation fictionnelle - qui fait l'objet d'un soin particulier de la part de l'auteur ${ }^{16}-$, on ne peut en dire autant pour les autres passages... Mais il est sûr que la comparaison est un moyen didactique remarquablement lisible dans les récits de voyage, et qu'instinctivement notre conscience littéraire nous pousse à associer métaphore et langue poétique, ce qui ne serait pas trahir Chateaubriand, qui revendique le nom de poème pour certaines de ses fictions narratives ${ }^{17}$. Or on rencontre le même phénomène chez Gautier :

\begin{tabular}{|c|c|}
\hline Un tour en Belgique & La Toison d'or \\
\hline $\begin{array}{l}\text { Dans la berline, les places sont } \\
\text { séparées comme des stalles de théâtre } \\
\text { [...]. (Caprices et zigzag dans } \\
\text { oruvres complètes, Paris / Genève, } \\
\text { Champion / Slatkine, } 1978, \text { p. } 65)\end{array}$ & $\begin{array}{l}\text { Tiburce s'assit dans sa stalle en } \\
\text { compagnie de cinq Wallons } \\
\text { immobiles à leurs places comme des } \\
\text { chanoines au chapitre [...] (Romans, } \\
\text { contes et nouvelles, P. Laubriet, éd, } \\
\text { Paris, Gallimard, } 2003, \text { t. I, p. } 779 \text { ) }\end{array}$ \\
\hline $\begin{array}{l}\text { [à la cathédrale d'Anvers] des } \\
\text { bouffées de colombes passai ent de } \\
\text { temps à autre et neigeaient en blancs } \\
\text { flocons sur la balustrade [...]. (ibid, p. } \\
\text { 72) }\end{array}$ & $\begin{array}{l}\text { [1e] clocher, que la neige de col ombes } \\
\text { dont il est recouvert en tout temps fait } \\
\text { ressembler à une aiguille des Alpes } \\
\text { (ibid, p. } 785 \text { ) }\end{array}$ \\
\hline Vouxge en Espagne & Militona \\
\hline $\begin{array}{l}\text { [à la corrida] Bientôt les choeurs des } \\
\text { cannes se jơgnent. aux vociférations } \\
\text { devenues insuffisantes. Les planchers } \\
\text { despalcos craquent et se fendent, et } \\
\text { la peinture des plafonds tombe en } \\
\text { pellicules blanchâtres comme une } \\
\text { neige entrem ẽlée de poussière. } \\
\text { (Voyage en Espagne, P. Berthier éd, } \\
\text { Paris, G allimar d } 1981, \text { p. } 122 \text { ) }\end{array}$ & $\begin{array}{l}\text { Les plaf onds craquaient sous les } \\
\text { admirations de l'étage supérieur, et la } \\
\text { peinture détachée s'envolait en } \\
\text { tourbillons de pellicules blanchâtres. } \\
\text { (Romans, contes ot nouvelles, op. cit, } \\
\text { t. I, p. 1154) }\end{array}$ \\
\hline $\begin{array}{l}\text { Quelques cyprès poussent à tr avers } \\
\text { les crevasses des mur ailles leurs noirs } \\
\text { soupirs de feudllage au milieu de toute } \\
\text { cette lumière et de tout cet azur, } \\
\text { comme une pensée triste dans la joie } \\
\text { d'une fête. (ibid, p. } 293 \text { ) }\end{array}$ & $\begin{array}{l}\text { [...] quelques cyprès séculaires } \\
\text { s'élevant silencieusem ent dans le bleu } \\
\text { du ciel, comme une pensée de } \\
\text { mélancolie au milieu de la joie. (ibid., } \\
\text { p. 1225) }\end{array}$ \\
\hline
\end{tabular}


21 Dans le premier exemple tiré du voyage en Belgique, la comparaison toute simple du récit de voyage est en quelque sorte intégrée au récit de fiction sous une forme métaphorique - qui évoque quelque catachrèse potentielle, en ces temps où, comme on le sait, le lexique ferroviaire n'est pas encore totalement inventé. Mais elle déclenche presque immédiatement une autre analogie, sans rapport aucun avec celle du récit de voyage, puisqu'elle joue sur un autre sens du mot stalle. Est-elle suscitée par cette sorte de syllepse de sens? Quelle est sa motivation dans le contexte narratif de la nouvelle? Dans le premier exemple issu du périple espagnol, on notera que la comparaison du récit de voyage, qui recherche manifestement une certaine exactitude du rendu impressif, est métaphorisée dans le roman, probablement par souci de cohérence avec la séquence descriptive à laquelle elle se rattache :

[1] À ce triomphe de la force morale sur la force brute, le peuple, saisi d'enthousiasme, éclata en transports frénétiques ; c'étaient des applaudissements, des cris, des trépignements à ne pas s'entendre; les amateurs secouaient à tour de bras les espèces de sonnettes et de tams-tams qu'ils apportent à la course pour émettre le plus de bruit possible. (Militona, op. cit., pp. 1153-1154)

Son thème est en effet contenu dans la première proposition (le peuple, saisi d'enthousiasme, éclata en transports frénétiques), et les deux autres se caractérisent par la présence de verbes et déverbaux d'action (applaudissements, cris, trépignements, secouaient).

Il faut toutefois rester prudent face à la tentation qui se fait jour d'associer la comparaison au récit de voyage et l'analogie à la fiction narrative, car les deux derniers exemples de chaque série semblent inverser la tendance en présentant des métaphores particulièrement recherchées dans les récits de voyage, ainsi que des comparaisons développées dans les formes romanesques. L'image du Tour en Belgique est d'une écriture beaucoup plus complexe que celle de La Toison d'or: outre la licence grammaticale - à forte connotation littéraire - qui use du verbe neiger dans un tour personnel, on constate la mise en commun de plusieurs sèmes (le mouvement; le déplacement d'air pour bouffées et colombes ; la blancheur pour colombes et neigeaient), le mot bouffées étant lui-même une émanation directe du co-texte descriptif :

[2] [...] de grandes vagues d'air nous baignaient la figure, et les frais baisers du vent séchaient sur nos fronts moites la sueur que la fatigue de l'ascension y avait fait perler [...]. (op. cit., p. 72)

On notera par ailleurs l'association du sème abstrait contenu dans bouffées, caractérisant l'inanimé, avec celui de colombes, concret et désignant un animé. On a le sentiment que, dans ce cas, la saisie immédiate de l'impression par l'écriture prime sur la logique, y compris syntaxique, de la formulation. On aura reconnu là un exemple prototypique? - d'écriture artiste ${ }^{18}$. On pourrait reprendre cette analyse pour les textes espagnols, en la simplifiant et en ajoutant que, dans ce cas, la métaphore (les cyprès poussent des soupirs) est de la stricte compétence du récit de voyage, et que la comparaison finale, absente du manuscrit, est donc un ajout - une surcharge ? postérieure au premier jet de l'écriture.

Qu'en déduire, si l'on considère ces exemples comme particulièrement représentatifs du corpus d'étude ? On a déjà évoqué la fonction pédagogique de la comparaison dans le récit de voyage, mais l'on peut ajouter que la métaphore est une forme d'analogie plus resserrée, donc plus "économique » dans un contexte fictionnel. Faut-il donc voir dans la métaphore en régime fictionnel la trace d'une écriture, sinon plus élaborée, du moins davantage contrainte par des modèles et des codes extérieurs à elle que celle du 
récit de voyage ? La relative liberté propre à celle-ci autoriserait du coup les auteurs à s'abandonner à des essais d'écriture, comme pourrait bien le manifester la tendance à l'expansion analogique dans les deux derniers exemples analysés, qui n'entreraient donc nullement en contradiction avec les autres.

\subsection{Homogénéité}

Si on laisse de côté maintenant la répartition entre comparaison et métaphore pour s'intéresser aux relations qui existent entre les comparants au sein de la microstructure descriptive ou de la macrostructure narrative, on rencontre là aussi des phénomènes de convergence tout à fait édifiants, et ce quelque forme que prenne l'analogie. Il suffit, pour s'en rendre compte, de reprendre celle, déjà citée, par laquelle Chateaubriand établit un parallèle entre le Nil et le Mississipi. Il s'agit, dans le Voyage en Amérique, d'une comparaison à vocation didactique, qui utilise un phénomène bien connu du lecteur (les crues du Nil) pour illustrer un phénomène a priori inconnu de lui (celles du Mississipi). La métaphore d'Atala est d'une tout autre portée; il suffit, pour s'en persuader, de reprendre la séquence qui se trouve en amont :

[3] Par intervalles, il élève sa voix, en passant sous les monts, et répand ses eaux débordées autour des colonnades des forêts et des pyramides des tombeaux indiens ; c'est le Nil des déserts. (op. cit., p. 34)

On voit en effet que l'analogie finale est préparée par les deux métaphores architecturales qui la précèdent (colonnades, pyramides) et qu'elle vient en quelque sorte couronner. Il y a donc un lien direct entre ces analogies, et un réseau de sens est créé : on a affaire à une métaphore filée, dont la fonction est au moins décorative.

On trouve un amusant exemple de métaphore filée chez le Gautier du voyage en Belgique :

\begin{tabular}{|c|c|}
\hline Un tour en Belgique & La Toison d'or \\
\hline 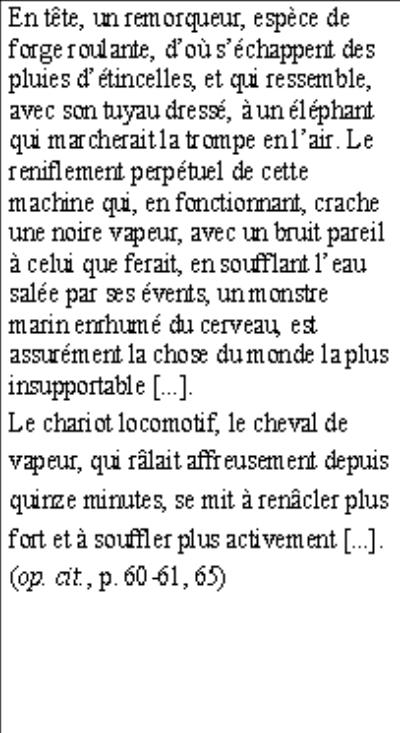 & $\begin{array}{l}\text { Le cheval de vapeur avait déjà mangé } \\
\text { son awoine de charbon, il renâclait } \\
\text { d'impatience et soufflait par ses } \\
\text { nase aux enflammés, avec un râle } \\
\text { strident, d'épaisses bouffées de fumée } \\
\text { blanche, entrem êlées d'aigr ettes } \\
\text { d'étincelles [...] bientôt le cheval } \\
\text { s'arima et fut pris d'une incr oyable } \\
\text { furie de vitesse. [...]C ependant le } \\
\text { monstre de fonte éructait avec un } \\
\text { bruit toujours croissant son haleine } \\
\text { d'eau bouillante ; il sifflait comme un } \\
\text { cachal ot asthmaticue, une sueur } \\
\text { ardente courrait ses flancs de bronze. } \\
\text { - Il semblait se plaindre de la rapidité } \\
\text { insensée de sa course et demander } \\
\text { grâce à ses noirs postillons qui } \\
\text { l'éperonnaient à gandes pelletées de } \\
\text { tourbe. (op. cit., p. } 779 \text {-780) }\end{array}$ \\
\hline
\end{tabular}

La confrontation entre ces deux séries d'extraits fait ressortir de manière frappante la différence de traitement réservée aux analogies. Dans le récit de voyage, on relève ainsi, sous une forme plutôt comparative (une espèce de, qui ressemble à, pareil à), une pléthore de comparants pour la locomotive, de la forge roulante (à laquelle se rattachent les pluies d'étincelles) au cheval de vapeur, qui râle ${ }^{19}$, renâcle et souffle, en passant par l' 
éléphant et le monstre marin. La nouvelle se concentre en revanche sur une seule métaphore, celle du cheval de vapeur, relayée par plusieurs autres, secondaires : avoine de charbon, il renâclait d'impatience et soufflait par ses naseaux enflammés, avec un râle strident, bientôt le cheval s'anima, la rapidité insensée de sa course, noirs postillons qui l'éperonnaient. Échappent seules à cette métaphore filée quelques analogies qui semblent inspirées de celles du récit de voyage: d'épaisses bouffées de fumée blanche, entremêlées d'aigrettes d'étincelles, le monstre de fonte éructait avec un bruit toujours croissant son haleine d'eau bouillante; il sifflait comme un cachalot asthmatique, une sueur ardente couvrait ses flancs de bronze. Encore peut-on hésiter à propos de certains comparants, qui partagent des sèmes avec celui de la métaphore filée, comme aigrettes - qui peut constituer un ornement hippique ${ }^{20}$ - ou flancs. A l'opposé de la dispersion sémantique repérée dans le récit de voyage, qui correspond à la nomination problématique de la locomotive, et que l'on attribuera certainement à la recherche d'un effet de comique hyperbolique, on ne peut que constater l'extrême cohésion sémantique de la métaphore filée dans $L a$ Toison d'or, cohésion à peine troublée par les allusions déjà citées, que l'on peut interpréter au choix comme une concession à l'esprit goguenard ou comme la volonté, manifeste chez Gautier, de ne pas laisser perdre un trait d'écriture.

\subsection{Efficacité narrative}

30 Mais la cohésion analogique existant au sein des descriptions en régime fictionnel, dans les textes de notre corpus du moins, dépasse peut-être la simple recherche ornementale, ou même celle d'une économie des effets narratifs : elle met en jeu des relations de solidarité, voire de motivation, entre la macrostructure narrative et la microstructure descriptive. Le choix de tel ou tel registre sémantique dans la création d'un réseau analogique à l'intérieur d'un système descriptif joue un rôle décisif dans la création du sens au plan du récit tout entier. La solidarité des comparants dans Paul et Virginie a déjà fait l'objet de diverses études ${ }^{21}$, et l'on a ailleurs tenté de mettre en lumière celle qui se manifeste dans Atala ou La Toison d'or ${ }^{22}$ : autant d'exemples de la prégnance de la macrostructure narrative au sein de la microstructure descriptive par le biais des comparaisons ou des métaphores, puisque celle-là va jusqu'à influencer les analogies en apparence les plus anodines de celle-ci. En régime fictionnel, les liens d'échange et de solidarité analogiques ont tendance à être beaucoup plus forts qu'il n'y paraît, tant au sein même de la séquence descriptive que dans les relations sémantiques qu'elle entretient avec le récit. La description se voit ici confirmée dans son rôle traditionnel de servante du récit.

31 Or, on n'a pu identifier de semblables cas de cohésion analogique dans les récits de voyage du corpus analysé. On a été frappé en revanche par la récurrence de certaines thématiques analogiques, qui reviennent de manière lancinante dans les comparants des récits de voyage d'une certaine ampleur, chez Chateaubriand ou Gautier. Dans une autre étude ${ }^{23}$, on s'est ainsi attaché aux multiples comparaisons géographiques renvoyant à des paysages italiens ou français dans l'Itinéraire. Si elles ont, à n'en pas douter, une valeur pédagogique pour le lecteur français qui ne connaît pas ces paysages, elles ne sont pas dépourvues non plus d'une valeur tantôt axiologique - le comparant dévalorise le paysage de référence et reflète la déception du voyageur tantôt mémorielle - les comparants italiens convoquent les souvenirs personnels de l'auteur, au même titre que les références au voyage en Amérique, et orientent le récit du périple oriental vers une dimension autobiographique. 
Tout se passe donc comme si certains comparants du récit de voyage, en se répétant de manière parfois obsédante, finissaient par créer horizontalement des réseaux de sens assez lâches, mais suffisamment présents pour être repérables, à l'opposé des relations majoritairement verticales entretenues, en régime fictionnel, par les analogies avec la macrostructure sémantique du récit. Le phénomène se vérifie dans le Voyage en Espagne de Gautier, où reviennent assez fréquemment des comparants culinaires ou minéraux. Les premiers peuvent s'expliquer par la constante préoccupation alimentaire de Théophile au cours de son voyage ${ }^{24}$, dont il a pu se souvenir en rédigeant son récit. Les seconds appartiennent, en apparence du moins, à une isotopie fréquente dans toute l'œuvre de Gautier, que certains critiques ont attribuée à ses préoccupations purement esthétiques comme à son souci de fixer dans une forme inaltérable une réalité qui courrait autrement le risque de perdre toute consistance ${ }^{25}$. Il faut noter toutefois que cette isotopie se concentre dans les pages du Voyage en Espagne consacrées à l'Andalousie, et en particulier à Grenade. Si l'on se souvient que Gautier parle à plusieurs reprises de cette ville comme du "paradis de [ses] rêves " ${ }^{26}$, faut-il voir dans ces analogies répétées la trace d'une opération de valorisation esthétique, destinée à rendre le paysage précieux, d'une sorte de "transmutation alchimique du monde, qui le vitrifie comme un émail, sans détruire la netteté de ses lignes ", pour reprendre la belle formule de J.-Cl. Berchet ${ }^{27}$ ? À coup sûr, l'horizon intertextuel des Mille et une Nuits n'est pas loin, jusque dans certains paysages délicatement érotisés :

[4] [...] la montagne semble avoir revêtu une immense robe de soie changeante, pailletée et cotelée d'argent [...] le diadème d'argent de la sierra étincelle encore dans la sérénité du ciel sous le baiser d'adieu du soleil. (Voyage en Espagne, op. cit., p. 266)

Récit de voyage et fiction narrative, s'ils ont l'un et l'autre abondamment recours aux figures de l'analogie, semblent donc en faire un usage notablement différent, comme tendent à le prouver les exemples que l'on vient d'analyser. Cette différence est-elle le signe d'un fonctionnement narratif dans lequel la description jouerait des rôles distincts, mais non absolument étrangers l'un à l'autre ? Une chose est sûre, c'est que les logiques de l'analogie dans ces deux genres sont difficilement compatibles, comme le montre la scène de la mise à mort du taureau dans Militona :

[5] L'épée, plantée au défaut de l'épaule, s'élevait avec sa poignée en croix entre les cornes du taureau et rappelait ces gravures gothiques où l'on voit saint Hubert à genoux devant un cerf portant un crucifix dans ses ramures.

L'animal s'agenouilla pesamment devant Juancho, comme rendant hommage à sa supériorité, et, après une courte convulsion, roula, les quatre sabots en l'air. (op. cit., p. 1156)

Elle est issue de deux notations relativement voisines du Voyage en Espagne:

[5'] Un éclair d'argent passa avec la rapidité de la pensée au milieu des deux croissants; le taureau tomba à genoux en poussant un beuglement douloureux, ayant la poignée de l'épée entre les deux épaules, comme ce cerf de saint Hubert qui portait un crucifix dans les ramures de son bois, ainsi qu'il est représenté dans la merveilleuse gravure d'Albert Dürer.

[...]

Le coup que venait de faire l'espada est, en effet, très estimé et se nomme la estocada a vuela pies : le taureau meurt sans perdre une goutte de sang, ce qui est le suprême de l'élégance, et en tombant sur ses genoux semble reconnaître la supériorité de son adversaire. (op. cit., p. 119-120)

Les visions successives du récit de voyage, par le biais des analogies, rendent compte avec précision des différents moments qui graduent la perception de la scène par le 
narrateur: dans la première, l'impression de rapidité, à travers les deux métonymies (un éclair d'argent passa [...] au milieu des deux croissants), la douleur du taureau, puis l'émotion esthétique, par le biais des deux analogies successives (le cerf de saint Hubert d'abord, puis la gravure de Dürer) ${ }^{28}$; dans la seconde, le jugement rationnel de l' aficionado, marqué par un discours argumentatif à visée axiologique ainsi que des précisions historiques et techniques.

Pourquoi donc ne peut-on s'empêcher d'éprouver un certain malaise devant l'extrait correspondant de Militona? Sans doute parce qu'il y a contradiction entre la visée impressive, étrangère au contexte romanesque, et le désir éprouvé par Gautier de montrer son savoir-faire littéraire... qui se manifeste dans cette accumulation un peu plaquée d'images étrangères au propos du roman. Selon P. Larthomas, "l'effet stylistique manqué est souvent plus révélateur que la trouvaille admirable ${ }^{29} \%$. Les extraits que nous venons d'analyser ne viennent-ils pas à l'appui de cette affirmation?

\section{Les enseignements d'une confrontation}

La confrontation des choix et des pratiques analogiques dans les quelques extraits ici sélectionnés, si elle mérite d'être confirmée par un corpus plus large, a tout de même permis de dégager un certain nombre de convergences dans l'usage de ce procédé caractéristique de la description en régime narratif. Qu'il s'agisse du privilège accordé à des métaphores frappantes, d'une tendance plus marquée à l'allégorie ou à la construction de réseaux de sens verticaux mettant la microstructure descriptive en relation directe avec la macrostructure narrative, les descriptions analysées dans un contexte fictionnel vont le plus souvent dans le sens de la concentration en vue d'une certaine efficacité narrative. Cette tendance est beaucoup moins nette dans les récits de voyage : les analogies descriptives, quand elles abandonnent la vertu pédagogique qui leur est propre, sont pour l'écrivain le lieu d'essais d'écriture qu'autoriseraient moins facilement les contraintes de la fiction. En outre, leur maillage sémantique parait, sinon plus lâche, du moins fondé sur la récurrence plus que sur la concentration, faute sans doute d'une structure narrative digne de ce nom. Or les autres phénomènes stylistiques étudiés au cours de cette recherche - longueur des descriptions, syntaxe, indices démarcatifs, travail du signifiant, indices de subjectivité, fonctions, distribution dans le récit - sont là aussi étonnamment convergents.

5.1. La description dans la fiction narrative : travaillée, motivée, asservie

Les fictions narratives examinées apparaissent en effet, quelles que soient leur diversité et leur inscription dans des genres fort hétérogènes en apparence, comme des discours fortement contraints, sinon pressés, dans leur relation à la description. La soumission $\mathrm{du}$ dispositif narratif tout entier à l'intrigue, la dramatisation de l'action, indispensables pour soutenir l'intérêt de la lecture, imposent le recours à une structure narrative forte et à un principe d'économie et de rentabilité qui laissent généralement peu de place à la digression ou au détail « insignifiant ». Cette soumission est d'autant plus forte que l'idéologie rhétorique du temps ne considère pas d'un œil bien favorable la description dans les genres fictionnels ${ }^{30}$.

Dans le même temps, on a le sentiment que ces ouvrages sont tenaillés par un souci toujours croissant d'individualiser action et personnages, de les insérer dans un cadre toujours mieux défini - en particulier lorsqu'il s'agit d'un cadre exotique, perçu comme tel ou dont l'originalité n'est pas toujours sensible au lecteur. S'y adjoint le sentiment que l'ouvrage plaira davantage si l'effet hallucinatoire est plus réussi et, partant, si la peinture de ce cadre est plus exacte, remplie de ces petits détails qui donnent l'illusion de la réalité et 
que seul le contact direct avec celle-ci par le biais du voyage est en mesure de transmettre. Ces tendances, auxquelles s'ajoute le respect de conventions esthétiques fortement ancrées dans l'horizon d'attente d'un certain public, semblent entraîner la description sur la pente inverse de l'expansion et du détail "révélateur", et l'amener à mettre en place les conditions pragmatiques d'une hypotypose ou d'une impression référentielle.

Dans ce cadre, les descriptions apparaissent comme des textes travaillés, motivés et asservis. Travaillés comme le montrent les procédés destinés à les insérer dans le récit ou, au contraire, à les en démarquer. Lorsque la description ne s'intègre pas à la syntaxe du récit, par le biais d'une pure et simple narrativisation, elle s'efforce de naturaliser ce qui fait sa différence, d'effacer les sutures qui risqueraient non plus de la mettre en valeur, mais de l'isoler au sein du récit et de souligner son caractère « rapporté ». Combien de personnages de roman ainsi mis en position de descripteurs potentiels par les nécessités de la narration et le biais d'une "thématique vide" finement analysée par Philippe Hamon? Telle description qui s'insère tout naturellement dans la continuité narrative d'un récit de voyage se retrouve comme mise en scène dans la fiction narrative par le recours à une séquence de récit destinée à placer le personnage contemplateur en un site qui justifie la qualité du paysage décrit et motive sa présence sur les lieux, ainsi que la pause narrative suscitée par cette description. Il s'agit d'une sorte de « récit postiche » privée de tout autre fonction que de justifier la présence du morceau descriptif à suivre dans le tissu narratif ${ }^{31}$.

41 Mais les descriptions en régime fictionnel se caractérisent également par leur motivation: d'étendue généralement plus limitée que dans les récits de voyage ${ }^{32}$, elles montrent une certaine propension à neutraliser - voire à éliminer purement et simplement - les discours non narratifs, considérés comme parasites, à homogénéiser leurs contenus, du point de vue syntaxique, sémantique et signifiant, et à les dramatiser de manière presque systématique. Ainsi, dans Paul et Virginie, les descriptions participent déjà pleinement de l'économie narrative, et entretiennent, avec la structure de l'œuvre, des relations d'échange qui les font sortir de la vocation purement décorative à laquelle les cantonnait, par précaution, la tradition rhétorique. Maints travaux ont mis en lumière les nombreux échos existant entre les représentations des paysages de l'île de France, dont le contenu est bien souvent emprunté au récit du voyage, et la macrostructure narrative du roman: états d'âme des personnages, évolution tragique de l'histoire, sens symbolique du récit ${ }^{33}$. À l'autre bout de la chronologie, Raymonde Debray Genette a bien montré comment Flaubert, empruntant certaines descriptions d'Un Coeur simple au récit de son voyage en Bretagne, éliminait dans la nouvelle toute trace de subjectivité, enthousiaste ou polémique, pour laisser «le système dramatique du conte parle[r] de lui-même $~^{34}$. Gautier ne procède pas autrement dans Militona.

Cette promotion de la description en régime fictionnel se double toutefois d'un asservissement au récit, jusque dans la structure de certaines d'entre elles, et surtout dans les fonctions qui leur sont imparties: elles peuvent alors servir de signal à un genre littéraire, de relais narratif, ou participer à l'élaboration tonale ou symbolique du récit, quitte à abdiquer au passage leur authenticité référentielle au profit du bon fonctionnement sémantique de celui-ci. Encore embryonnaire chez Bernardin, cette «rentabilisation» de la description, qui va de pair avec une concentration aux points névralgiques du récit, semble se renforcer dans les ouvrages de Chateaubriand, et paraît un fait tellement établi chez Gautier que celui-ci, dans la perspective excentrique qui est souvent la sienne, n'hésite pas à en dénuder les procédés pour mieux les dénoncer. Ainsi la représentation d'un jardin grenadin dans Militona, sorte de condensé des impressions du 
voyage en Andalousie, se présente comme le symbole explicite du bonheur des héros enfin mariés, et qui habitent la demeure attenante :

[6] Tout homme, en passant par cette rue et en jetant l'œil dans cet intérieur, quelque mauvais observateur qu'il fût, n'eût pu manquer de dire : «Là vivent des gens heureux. » Le bonheur illumine les maisons et leur donne une physionomie que n'ont pas les autres. Les murailles savent sourire et pleurer; elles s'amusent ou elles s'ennuient; elles sont revêches ou hospitalières, selon le caractère de l'habitant qui leur sert d'âme : celles-ci ne pouvaient être animées que par de jeunes amants ou de nouveaux époux. (op. cit., p. 1225)

De la même manière, le paysage désolé des environs de Madrid, qui fait l'objet de plusieurs descriptions apparentées dans le Voyage en Espagne, est utilisé dans le roman pour souligner l'état d'âme d'un personnage délaissé par celle qu'il aime et qui se retrouve à errer «au hasard à travers la campagne. » La description s'achève par ces mots :

[7] [...] les pentes et les collines n'offrent aucune verdure et forment un paysage en harmonie avec tous les sentiments tristes. La gaieté s'y éteindrait, mais au moins le désespoir ne s'y sent raillé par rien. (ibid., p. 1215)

La surenchère interprétative, dans un sens comme dans l'autre, ne fait que souligner le caractère suranné de ces deux clichés que sont désormais devenus le locus amœnus et le locus horridus : Saint-Lambert ne conseillait-il pas déjà, en son temps, de les mettre en rapport avec l'état d'âme des personnages ${ }^{35}$ ? Autant de clins d'œil au lecteur de la part d'un Gautier point dupe de ces procédés traditionnels du roman romantique...

5.2. La description en récit de voyage : victime ou agente de son autonomie?

Les récits de voyage du corpus sélectionné font preuve en revanche d'une moindre consistance narrative, accueillant, sans nécessairement les unifier, des formes de discours aussi variées qu'hétérogènes, et fondant leur intérêt sur une certaine attitude à l'égard du réel approché. La diversification des publics concernés et le fait que des écrivains deviennent, au tournant du siècle, les régisseurs discursifs d'un genre qui se voulait à l'origine essentiellement tourné vers le savoir, amènent deux difficultés supplémentaires, qui risquent en permanence d'entraîner les récits de voyage sur la voie de la dispersion, de la redite, de la falsification et de la demi-mesure. Rien d'étonnant à ce que nombre d'écrivains, à l'instar de Flaubert, prétendent «le genre voyage [...] par soi-même une chose presque impossible. $\|^{36}$

Dans ce cadre très particulier, la description se trouve bien entendu en première ligne : pièce essentielle du dispositif cognitif représenté par le récit de découverte et d'exploration traditionnel, elle était jusqu'alors cantonnée par celui-ci à un rôle purement informatif, qui finit par embarrasser les écrivains, conscients des attentes parfois contradictoires de leur public. Ils adoptent le plus souvent une attitude ambiguë à l'égard du didactisme et de l'érudition, toujours susceptibles de leur attirer les foudres d'une critique sourcilleuse et habile à repérer erreurs et lacunes, mais aussi de susciter l'ennui ou les redites et de leur aliéner ainsi un lectorat plus avide d'impressions et de sincérités ${ }^{37}$. La représentation du paysage leur offrira d'ailleurs un soutien précieux pour affirmer la nouveauté et l'originalité de leur projet en matière de récit de voyage et de description. Chateaubriand oppose ainsi sa démarche à celle des explorateurs qui l'ont précédé, dans la préface de l'Itinéraire:

Je prie donc le lecteur de regarder cet Itinéraire, moins comme un Voyage que comme des Mémoires d'une année de ma vie. Je ne marche point sur les traces des Chardin, des Tavernier, des Chandler, des Mungo Parck, des Humboldt : je n'ai point la prétention d'avoir connu des peuples chez lesquels je n'ai fait que passer. Un 
moment suffit au peintre de paysage pour crayonner un arbre, prendre une vue, dessiner une ruine; mais les années entières sont trop courtes pour étudier les mœurs des hommes, et pour approfondir les sciences et les arts. (op. cit., p. 701) récits de voyage examinés, assez "détendues ", à tous les sens du terme, puisque, plus lâchement encadrées qu'en régime fictionnel et ayant de ce fait une nette propension à s'étendre et à absorber toutes sortes de discours, elles doivent recourir à des procédures internes ou externes en mesure de les structurer ou de les signaler comme telles, en particulier comme morceaux d'ekphrasis, c'est-à-dire comme œuvres d'art. Elles se voient ainsi émaillées d'innombrables références à la peinture, au point qu'on a pu parler d'un véritable «code pictural romantique $»^{40}$, qui contribue sans doute à affirmer la nature profondément originale et artistique des voyages d'écrivains au XIX siècle.

Mais peut-être ces descriptions gagnent-elles justement en autonomie ce qu'elles perdent en efficacité. Ces incessantes références picturales ne les empêchent pas de faire fi de la tradition de l'ut pictura poesis - qui entendait les réduire à tout prix à des sortes de "peintures écrites" - pour adhérer pleinement à la linéarité de leur déroulement textuel et, partant, conduire l'auteur comme le lecteur sur les voies inexplorées de la recherche impressive ou esthétique comme de l'univers mental et imaginaire de l'écrivain, sur lesquelles les fictions narratives éprouvent souvent quelque peine à s'avancer ${ }^{41}$.

De la même manière, la description apprend à retourner à son avantage les inévitables répétitions qu'elle doit affronter dans le récit de voyage et qui constitue bien souvent le cauchemar de l'écrivain scrupuleux ${ }^{42}$. Substituant la dispersion et surtout la récurrence descriptives aux procédures de concentration et d'efficacité narratives qui marquent la fiction, la description peut élaborer, au fil des récits de voyage, des réseaux de sens certes moins serrés, mais non moins efficaces, on l'a vu ${ }^{43}$. La faiblesse du tissu narratif proposé par la simple cohérence spatio-temporelle de l'itinéraire souligne la variété, mais aussi la disparité des discours qui traversent les récits de voyage. Or l'écrivain ne peut négliger le poids des contraintes idéologiques, en particulier celui de la doxa littéraire, qui place à l'horizon de l'écriture, mais aussi de la lecture, l'idée même de composition. Cela amène parfois les écrivains voyageurs à des choix formels non dénués de paradoxes, des hésitations de Bernardin entre la lettre, le journal et la taxinomie scientifique, au mélange des formes qui marque le Voyage en Amérique ou le Voyage en Italie de Chateaubriand. Mais la description sait également jouer des ruptures liées à la juxtaposition des séquences et des discours dans les récits de voyage, pour en tirer parfois une certaine force argumentative, chez Bernardin, Chateaubriand ou Flaubert, dénonçant les horreurs de l'esclavage, la cupidité de la tyrannie ottomane ou la bêtise des bourgeois. À d'autres moments, ces absences de transition peuvent contribuer au renforcement du genre - comme cela est manifeste dans le Voyage en Amérique du même Chateaubriand, qui en fait l'un des piliers de la rhétorique du naturel qu'il déploie dans sa description fictive des Florides - ou à sa mise en question, comme chez le Gautier du 
Tour en Belgique qui, à coups d'« à propos ", court-circuite avec désinvolture un récit où, selon le mot de Flaubert, la principale difficulté réside précisément «dans les transitions $»^{44}$.

51 Face aux carences structurelles du récit de voyage, la description peut aussi en venir à assumer la direction des opérations narratives, à l'échelle d'un fragment, comme c'est le cas dans le "Journal sans date" du Voyage en Amérique, ou dans la "Lettre à Fontanes » du Voyage en Italie de Chateaubriand, que son auteur présente certes comme un «monceau de ruines» ou un "énorme fatras ${ }^{45}$ de paysages et de monuments juxtaposés, mais qui se révèle à l'examen savamment composée ${ }^{46}$. La description peut aussi jouer ce rôle de manière plus souterraine, à l'échelle du récit tout entier. Dans une certaine mesure en effet, si le récit de surface juxtapose une série d'événements et d'objets sans autre lien pour les réunir que le parcours à la fois chronologique et géographique, il semble régi en profondeur par des réseaux narratifs plus logiquement organisés, fondés sur l'analogie et la récurrence de certains motifs. L'Itinéraire peut se présenter ainsi comme la reconstitution d'un pèlerinage à Jérusalem où se succèdent les étapes symboliques, où le moi prend conscience de sa dimension temporelle à travers la confrontation avec l'expérience américaine ou les variations sur un même paysage ${ }^{47}$. Dans le Voyage en Espagne de Gautier, on assisterait en revanche à la libération du moi et de l'écriture à travers la confrontation avec l'art et la réalité " exotique ${ }^{48}$. Or ces réseaux se constituent moins sur une base narrative que sur la récurrence et l'articulation d'une série de descriptions. Ainsi, en dépit de la dispersion et du désordre apparents qui les caractérisent, ces récits de voyage sont en réalité profondément structurés, et la description joue dans ce cadre un rôle essentiel ${ }^{49}$.

52 Cette forme de prise du pouvoir par la description au bénéfice d'un récit de voyage qui y gagne une structure narrative, semble correspondre à l'évolution même du genre. Mais elle ne relève peut-être après tout que du désir fort commun

que les événements réels offrent la cohérence, l'intégrité, la complétude et la clôture d'une image de la vie qui ne peut être qu'imaginaire ${ }^{50}$.

53 Mais cela ne justifie pas pour autant que l'on fasse de la narration au sein du récit de voyage la servante de la description: la démarche logique reste indéniablement narrative, puisqu'elle s'attache à décrire une évolution, un parcours psychologique ou personnel. Pour reprendre les mots utilisés par Gérard Genette au sujet du roman contemporain, cette "promotion spectaculaire de la fonction descriptive» n'en confirme pas moins de manière flagrante « son irréductible finalité narrative $»^{51}$. Pour reprendre les mots d'Adrien Pasquali,

s'il est généralement admis [...] que dans le mode romanesque le descriptif est soumis aux impératifs du narratif, il n'est pas du tout avéré qu'il en aille différemment pour le récit de voyage, d'une manière à la fois plus avertie et plus masquée ${ }^{52}$.

On se gardera donc de voir trop vite dans la servante du roman et de ses avatars la maitresse absolue du récit de voyage...

Il n'en reste pas moins que l'on assiste indubitablement, dans la période choisie, au passage d'un récit de voyage de découverte et d'aventure, dans lequel le monde extérieur et sa (re)connaissance seraient l'enjeu principal, au récit d'une expérience, qui place l'individu voyageur au centre de ses préoccupations ${ }^{53}$.

Il est aussi certain que l'on retrouve dans les textes étudiés quelques-uns des axes privilégiés sur lesquels tous les écrivains qui chercheront à explorer les voies nouvelles 
de l'autobiographie, romancée ou non, s'aventureront à leur tour : on sait le rôle joué par la description de paysage dans le roman romantique ${ }^{54}$. Et l'on ne pourrait pas nier davantage qu'en raison de sa plus grande liberté, la description offre en récit de voyage un terrain plus favorable à l'exploration du moi qu'en régime fictionnel : cela explique peut-être l'adieu à la fiction narrative que Chateaubriand place en conclusion des Martyrs et qu'il renouvelle à la fin de l'Itinéraire ${ }^{55}$.

Pour toutes ces raisons, la frontière entre les deux genres étudiés, nettement établie au plan de la réception, a tendance, au fil du siècle, à se faire toujours plus floue au plan de l'écriture, et l'on assiste indéniablement à des échanges de procédés entre récit de voyage et fiction narrative: rhétorique de la démarcation descriptive, phénomènes d'intertextualité énonciative, mise à distance ironique de la représentation, entre autres. Ces échanges sont-ils l'indice que les frontières entre récit de voyage et fiction narrative sont progressivement en train de s'estomper? On sait que ces deux genres ont pour point commun leur capacité à intégrer et à absorber des formes de discours extrêmement variées ${ }^{56}$, ce qui n'est peut-être pas étranger à leur succès auprès du public et surtout des auteurs romantiques, peu soucieux de rétablir des frontières génériques étanches qu'ils ont systématiquement malmenées. Mais il est aussi évident qu'un ouvrage comme le Voyage en Orient de Nerval, ou beaucoup plus tard, les romans et récits de voyage de Loti, à forte dominante autobiographique, et dans lesquels l'exploration du moi et de sa sensibilité jouent le rôle que l'on sait, laissent planer, en dehors de leur paratexte, bien des doutes sur leurs positions à l'égard de la fiction, et ouvrent peut-être la route aux recherches contemporaines en la matière. On n'a pas encore tenté de confrontation en la matière : que ne révélerait-elle pas?

Autant de perspectives que cette étude entendait simplement suggérer. On espère juste avoir esquissé des éléments de réponse à la question posée au début de cette étude : existe-t-il des "stylèmes génériques ", autrement dit des indices stylistiques susceptibles de caractériser un genre littéraire? Bien entendu, la faible étendue de la présente étude, et surtout le corpus encore limité sur lequel elle se fonde, ne nous permettent pas à ce stade de clore la question, loin s'en faut. Mais on a tout de même pu constater qu'un auteur pouvait faire usage de motifs, voire de procédés semblables dans des genres littéraires différents, mais que ces motifs et ces procédés communs étaient alors utilisés de manière différente en fonction des genres abordés, et que leur confrontation pouvait apporter des éléments d'information importants et parfois inattendus sur les contraintes intrinsèques à ces genres, contraintes qui sont souvent loin d'être explicites. Ce n'est donc pas seulement l'intuition de Pierre Larthomas qui se voit ici confirmée, ce sont aussi celles de nombreux poéticiens des genres littéraires complexes que sont la fiction narrative et le récit de voyage. Gageons qu'une étude plus étendue dans la chronologie et dans la gamme des genres confrontés apporterait encore bien des surprises à une stylistique des genres qui ne demande qu'à être refondée. 


\section{BIBLIOGRAPHIE}

Adam J.-M. (1993). La Description. Paris : PUF.

Adam J.-M. \& Petitjean A. (1989). Le Texte descriptif. Paris : Nathan.

Berchet J.-Cl. (1981). « Introduction », in Th. Gautier, Voyage en Espagne. Paris : Garnier-

Flammarion.

Berchet J.-Cl. (1983). « Un voyage vers soi », Poétique 53 : 91-108.

Berchet J.-Cl. (1994). « La préface des récits de voyage au XIX ${ }^{\mathrm{e}}$ siècle », in G. Tverdota (éd.), Ecrire le voyage. Paris: Presses de la Sorbonne nouvelle, 3-15.

Bulgin K. (1988). The Making of an Artist. Gautier's Voyage en Espagne. Birmingham (Alabama) : Summa.

Cogard K. (2001). Introduction à la stylistique. Paris : Flammarion.

Combe D. (1992). Les Genres littéraires. Paris : Hachette.

Debray-Genette R. (1988). Métamorphoses du récit. Paris : Le Seuil.

Dubois J. (1963). Romanciers français de l'instantané au XIXe siècle. Bruxelles : Palais des Académies.

Frappier-Mazur L. (1980). « La description mnémonique dans le roman romantique », Littérature $38: 3-26$.

Gardes-Tamine J. (1992). La Stylistique. Paris : Colin.

Genette G. (1969). Figures II. Paris : Le Seuil.

Genette G. (1991). Fiction et diction. Paris : Le Seuil.

Guyot A. (1996-a). « Une certaine manière de voir ». La description entre récit de voyage et récit de fiction chez Bernardin de Saint-Pierre, Chateaubriand et Théophile Gautier. Essai de confrontation stylistique. Thèse de doctorat soutenue en 1996 à l'Université de Paris-Sorbonne.

Guyot A. (1996-b). « Libertés factuelles et contraintes fictionnelles : les descriptions américaines de Chateaubriand et leur mise en récit dans Atala, Les Natchez et le Voyage en Amérique ", Bulletin de la Société Guillaume-Budé 54, 4 : 357-372.

Guyot A. (1997). « Théophile et le 'cheval de vapeur'. Le premier voyage ferroviaire de Gautier (Belgique, 1836) », in Fr. Moureau \& M.-N. Polino (éd.), Ecritures du chemin de fer. Paris :

Klincksieck, 49-57.

Guyot A. (1998). « ‘Ce monceau de ruines...' : sens et fonctions des descriptions dans la lettre à Fontanes sur la campagne romaine », Bulletin de la Société Guillaume-Budé 56, 1 : 87-106.

Guyot A. (1999). «L'Orient de Chateaubriand : Florides ou Bourbonnais ? Sens et fonction des analogies dans l'Itinéraire de Paris à Jérusalem », in Chr. Montalbetti (éd.), Chateaubriand. La fabrique du texte. Rennes : PUR, 73-88.

Guyot A. (2003). « Le jardin-paysage de Paul et Virginie : transplantation textuelle ou alchimie littéraire ?», in B. Sosien (éd.), L'imaginaire du jardin. Cracovie : Univ. Jagellone, 134-146.

Hamon Ph. (1972). «Qu'est-ce qu'une description?», Poétique 12 : 465-485.

Hamon Ph. (1981). Introduction à l'analyse du descriptif. Paris : Hachette. 
Hamon Ph. (1991). La Description littéraire. Paris : Macula.

Ibsch E. (1982). « Historical Changes of the Function of Spatial Description in Literary Texts », Poetics Today 3-4 : 97-113.

Lafon H. (1982). « Sur la description dans le roman du XVIII e siècle », Poétique 51 : 303-313.

Larthomas P. (1964). « La notion de genre littéraire en stylistique », Le Français moderne 32 :

185-193.

Larthomas P. (1998). Notions de stylistique générale. Paris : PUF.

Le Huenen R. (1987). « Le récit de voyage : l'entrée en littérature », Etudes littéraires 20-1 : 45-57.

Legros G. (1976). « Description, la mal-aimée », Cahiers d'analyse textuelle 18 : 107-121.

Magri V. (1996). « Les enjeux pragmatiques du récit de voyage », Travaux du Cercle linguistique de Nice $18: 17-34$.

Magri V. (2005). « Hétérogénéité, cohésion et cohérence : le statut de l'anecdote digressive dans un récit de voyage », in A. Jaubert (éd.), Cohésion et cohérence. Paris : ENS Editions, 157-173.

Mallétroit G. (1986). « Les récurrences et les annonces dans Paul et Virginie », in J.-M. Racault (éd.), Etudes sur Paul et Virginie et l'œuvre de Bernardin de Saint-Pierre. Saint-Denis de la Réunion / Paris : CRLH / Didier, 119-159.

Mauzi R. (1966). «Préface », in B. de Saint-Pierre, Paul et Virginie. Paris : Garnier-Flammarion.

Molinié G. (1986). Eléments de stylistique française. Paris : PUF.

Molinié G. (1993). La Stylistique. Paris : PUF.

Molino J. (1992). « Logiques de la description », Poétique 91 : 363-382.

Montalbetti Chr. (1997), Le Voyage, le monde et la bibliothèque. Paris : PUF.

Mylne V. (1981). The $18^{\text {th }}$ Century French Novel, Techniques of Illusion. Cambridge : University Press.

Pasquali A. (1994). Le Tour des horizons. Critique et récits de voyage. Paris : Klincksieck.

Quéfellec L. (1988). « La construction de l'espace exotique dans le roman d'aventures au XIX ${ }^{\mathrm{e}}$ siècle ", in A. Buisine \& N. Dodille (éd.), L'Exotisme. Paris : Didier, 353-364.

Racault J.-M. (1986-a). « Ouverture et clôture dans Paul et Virginie », in J.-M. Racault (éd.), Etudes sur Paul et Virginie et l'œuvre de Bernardin de Saint-Pierre. Saint-Denis de la Réunion / Paris : CRLH / Didier, 83-102.

Racault J.-M. (1986-b). « Système de la toponymie et organisation de l'espace romanesque dans Paul et Virginie ", Studies on Voltaire and the 18th Century 242 : 377-418.

Racault J.-M. (1986-c). « Virginie entre la nature et la vertu. Cohésion narrative et contradictions idéologiques dans Paul et Virginie », Dix-Huitième Siècle 18 : 389-404.

Sangsue D. (1987). Le Récit excentrique. Paris : Corti.

Schapira M.-Cl. (1983). « Le langage de la couleur dans les nouvelles de Théophile Gautier », in Cl. Lacoste (éd.), Théophile Gautier, l'Art et l'Artiste. Montpellier : Soc. Théophile Gautier, 269-279.

Vissière J.-L. (1986). « Une page apocalyptique de Paul et Virginie », in J.-M. Racault (éd.), Etudes sur Paul et Virginie et l'œuvre de Bernardin de Saint-Pierre. Saint-Denis de la Réunion / Paris : CRLH / Didier, 36-39. 


\section{NOTES}

1.. Voir aussi Combe (1992: 103-107).

2.. Voir à ce sujet les analyses de Lafon (1982: 309-310).

3.. On mettra à part le cas de Par les champs et par les grèves, récit du voyage en Bretagne réalisé par Flaubert et Du Camp en 1847 : le texte a été rédigé « à quatre mains » en vue d'une publication qui n'aura jamais lieu du vivant de l'auteur.

4.. Genette (1991: 91).

5.. Genette $(1991: 60)$.

6.. Sangsue (1987:56)

7.. Voir à ce sujet Quéffelec (1988: 353-358).

8.. Voir les titres de Ph. Hamon, J.-M. Adam, A. Petitjean cités en bibliographie, ainsi que la controverse engagée par J. Molino.

9.. En dehors des propositions de Hamon (1981: 130-32), on peut aussi se référer aux travaux de G. Legros, E. Ibsch, J. Gardes-Tamine et G. Molinié.

10.. Voir à ce sujet Hamon (1981: 185-187).

11.. Voir en particulier Molinié (1986 : $164-166$ et $174-177 ; 1993: 32-36)$.

12.. (1964: 187).

13.. Hamon (1981: 66 et 168) (entre autres).

14.. Voir Magri (1996: $20 \mathrm{sq})$.

15.. Voir entre autres Montalbetti (1997: $176 s q)$.

16.. «Chactas, l'amant d'Atala, est un Sauvage, qu'on suppose né avec du génie, et qui est plus qu'à moitié civilisé, puisque non seulement il sait les langues vivantes, mais encore les langues mortes de l'Europe. Il doit donc s'exprimer dans un style mêlé, convenable à la ligne sur laquelle il marche, entre la société et la nature. Cela m'a donné de grands avantages, en le faisant parler en Sauvage dans la peinture des mœurs, et en Européen dans le drame et la narration. Sans cela il eût fallu renoncer à l'ouvrage : si je m'étais toujours servi du style indien, Atala eût été de l'hébreu pour le lecteur. »(Préface d'Atala, op. cit., p. 20).

17.. Voir ibid., p. 18 ; note des Natchez dans Euvres romanesques et voyages, op. cit., t. I, p. 578 ; Préface et Examen des Martyrs, op. cit., pp. 37-40 et 91-92.

18.. Voir Dubois (1963 : $93 \mathrm{sq}$ ) ; Gardes-Tamine (1992 : 67-68).

19.. Le mot s'emploie en effet pour désigner le cri de certains animaux.

20.. À moins que l'on ne considère le terme sous un jour strictement dénotatif (aigrette est aussi utilisé pour désigner des phénomènes naturels empruntant la forme d'une aigrette) : il ne s'agirait plus dans ce cas d'une analogie, mais d'une catachrèse.

21.. Voir par exemple Mauzi (1996 : 15) ; Mylne (1981 : 251) ; Vissière (1986) ; Mallétroit (1986) ; Guyot (2003).

22.. Voir Guyot (1996a et 1997).

23.. Guyot (1999).

24.. Il déclare par exemple au cours du voyage de Madrid à Grenade : "C'est à peu près vers cet endroit que j'ai, pour la première fois de ma vie, véritablement souffert de la faim » (Voyage en Espagne, op. cit., p. 242).

25.. Voir Schapira (1983 : 275-277).

26.. Voyage en Espagne, op. cit., p. 241; cf. aussi p. 228, 245, 253.

27.. Berchet (1981: 35). 
28.. On notera au passage la préparation, dans la première vision, de l'allusion à Dürer par une discrète isotopie d'ordre héraldique (argent / croissants) et la mise en scène de la vision de Gautier en quelque sorte placée en abyme par l'évocation de saint Hubert.

29.. Larthomas (1964: 190).

30.. « [...] la description, à moins qu'il ne s'agisse de récits de voyage, ne doit pas faire le fond d'un ouvrage, mais seulement en être l'ornement [...]. » (Pierre Larousse, article « Descriptif », Grand Dictionnaire universel du XIX ${ }^{e}$ siècle, 1866-1876). Voir Hamon (1981: 8 $s q)$.

31.. Voir Hamon (1981 : 185-186).

32.. Une brève étude statistique sur le corpus étudié - soit environ deux cents confrontations, tous auteurs et œuvres confondus - nous a permis d'établir que cette hypothèse se vérifiait dans quatre-vingt-dix pour cent des cas.

33.. Voir en particulier les études que J.-M. Racault a consacrées au prologue du roman (1986-a et b) et à la description de la canicule qui précède le premier ouragan (1986-c) ; voir aussi J.-L. Vissière pour une autre analyse de cet épisode.

34.. Debray-Genette (1988: $248 s q)$.

35.. « On doit assortir les épisodes aux paysages. / Il y a de l'analogie entre nos situations, les états de notre âme, et les Sites, les phénomènes, les états de la Nature. / Placez un malheureux dans un pays hérissé de rochers, dans de sombres forêts, auprès des torrents, etc. ; ces horreurs feront une impression qui se confondra avec celle de la pitié. / Placez de jeunes gens amoureux sous de riants berceaux, sur des fleurs, dans un pays heureux, sous un ciel pur et serein, etc., les charmes de la Nature ajouteront au sentiment voluptueux qu'inspirent les tableaux de l'amour » (Discours préliminaire aux Saisons, cité dans Philippe Hamon 1991 : 71).

36.. Flaubert, lettre à Taine, fin nov. 1866 (Les CEuvres de Gustave Flaubert. Lausanne : Club de l'Honnête Homme, 1965, t. XII, p. 156). Voir Le Huenen.

37. Voir les atermoiements de Chateaubriand à ce sujet dans l'Itinéraire.

38.. Berchet (1994: 10-12).

39.. On trouve le même genre d'analyse chez Gautier (Voyage en Espagne, op. cit., p. 148 et 307) et Custine (L'Espagne sous Ferdinand VII, J.-F. Tarn éd., Paris : Bourin, p. 16).

40.. La formule est de Berchet (1981 : 34) ; voir également Berchet (1994).

41.. Voir Guyot (1996b : 365 sq).

42.. « Pour que le volume n'eût aucune répétition, il aurait fallu vous abstenir de dire ce que vous aviez vu » (Flaubert, lettre à Taine, loc. cit.).

43.. V. Magri a proposé une analyse très voisine à propos des anecdotes en récit de voyage (2005: $168 s q)$.

44.. Lettre du 3 avril 1852 (Les CEuvres de Gustave Flaubert, op. cit., t. VI, p. 248).

45.. Euvres romanesques et voyages, op. cit., t. II, p. 1491 et 1496.

46. Voir Guyot (1998).

47. Voir Berchet (1983).

48. Voir Berchet (1981 : 51-53) ; Bulgin (1988).

49.. Voir Sangsue (1987 : 355).

50.. Pasquali (1994 : 47).

51.. Genette (1969: 59).

52.. Pasquali (1994 : 94).

53.. Ibid. : 91

54.. Voir Frappier-Mazur (1980).

55.. Op. cit., pp. 482-483 et 1214 . 


\section{RÉSUMÉS}

Stylèmes et corpus génériques : un essai de confrontation au service de la stylistique des genres Existe-t-il des indices susceptibles de caractériser un genre littéraire du point de vue stylistique? La confrontation, chez un même écrivain, de textes de référent identique, mais dans des genres littéraires différents, serait susceptible d'apporter des éléments de réponse à cette brûlante question, naguère posée par Pierre Larthomas. Mais ces éléments ne pourront être utilement recueillis que si l'on procède à une sélection rigoureuse et attentive du corpus de référence, du corpus d'étude et des outils d'investigation. On proposera ici un exemple de confrontation : les descriptions issues des voyages qu'un certain nombre d'écrivains français ont réalisés entre le tournant des Lumières et la fin du XIX ${ }^{e}$ siècle, descriptions que l'on retrouve en particulier dans les œuvres narratives fictionnelles et les récits de voyage de ces mêmes écrivains. On étudiera entre autres l'usage d'un procédé particulièrement significatif dans ce contexte : les figures de l'analogie.

Stilemi e corpus generici : un tentativo di confronto al servizio della stilistica dei generi Chi s'interessa alla stilistica dei generi letterari si può chiedere se ci sono delle marche caratteristiche, per ogni genere, dal punto di vista stilistico. Per uno stesso autore, il confronto fra testi dedicati allo stesso referente, in generi letterari diversi, può fornire elementi di rilievo per una risposta a questo difficile argomento proposto, tempo fa, da Pierre Larthomas. Bisogna però procedere ad una selezione molto attenta del corpus di riferimento, di quello di ricerca, nonché degli strumenti d'indagine. Questo studio sarà dedicato ad un esempio di confronto: quello delle descrizioni ispirate dai viaggi compiuti da diversi scrittori francesi fra la fine del '700 e la fine dell'800, descrizioni che si ritrovano sia nella narrativa di finzione sia nei racconti di viaggio di questi autori. Si darà particolare rilievo ad uno schema significativo: quello dell'analogia e del suo uso.

\section{INDEX}

Mots-clés : stylistique, genre littéraire, stylème, description, comparaison, métaphore

\section{AUTEUR}

\section{ALAIN GUYOT}

Université Stendhal, Grenoble 3 